\title{
Existence Results for Fractional Differential Equations with Multistrip Riemann-Stieltjes Integral Boundary Conditions
}

\author{
Tongling Lv, ${ }^{1}$ Huihui Pang $\left(\mathbb{D},{ }^{1}\right.$ and Limei $\mathrm{Cao}^{2}$ \\ ${ }^{1}$ College of Science, China Agricultural University, Beijing 100083, China \\ ${ }^{2}$ School of Mathematics and Physics, University of Science and Technology Beijing, Beijing 100083, China \\ Correspondence should be addressed to Huihui Pang; phh2000@163.com
}

Received 29 December 2017; Revised 28 February 2018; Accepted 20 March 2018; Published 24 April 2018

Academic Editor: Chris Goodrich

Copyright (c) 2018 Tongling Lv et al. This is an open access article distributed under the Creative Commons Attribution License, which permits unrestricted use, distribution, and reproduction in any medium, provided the original work is properly cited.

\begin{abstract}
This paper is concerned with the existence and multiplicity of the positive solutions for a fractional boundary value problem with multistrip Riemann-Stieltjes integral boundary conditions. Our results are based on the Leggett-Williams fixed point theorem. In the end, two examples are worked out to illustrate our main work.
\end{abstract}

\section{Introduction}

Nowadays, differential equations with fractional order have gained much attention and importance since they provided valuable tools for their applications in various sciences, such as gas dynamics, nuclear physics, electrodynamics of complex medium, and polymer rheology. With this advantage, fractional order models are regarded as more realistic and practical. For more details about fractional differential equations, we refer the readers to the monographs [1-4] and papers [5-9].

Many scholars have studied the existence of nonlinear fractional differential equations with a variety boundary conditions. However, it is better to impose nonlocal conditions because they can accurately describe the actual phenomenon. Some authors studied multipoint boundary value problems; for example, [10] discussed the infinite-point boundary value problems

$$
\begin{aligned}
D_{0+}^{\alpha} u(t)+f(t, u(t)) & =0, \quad t \in(0,1), \\
u(0) & =D_{0+}^{\beta} u(0)=0, \\
D_{0+}^{\beta} u(1) & =\sum_{i=1}^{\infty} \xi_{i} D_{0+}^{\beta} u\left(\eta_{i}\right),
\end{aligned}
$$

where $2<\alpha \leqslant 3,1 \leqslant \beta \leqslant 2,1 \leqslant \alpha-\beta, 0<\xi_{i}, \eta_{i}<1$ with $\sum_{i=1}^{\infty} \xi_{i} \eta_{i}^{\alpha-\beta-1}<1$. Existence result of at least two positive solutions is given via fixed point theorem in a cone.
Different from [10], some work focuses on the solvability of the fractional differential equations with integral boundary conditions. The details are found in [11-16] and the references therein. In [17], Sun and Zhao investigated the following fractional differential equation with integral boundary conditions:

$$
\begin{aligned}
D_{0+}^{\alpha} u(t)+q(t) f(t, u(t)) & =0, \quad t \in(0,1), \\
u(0) & =u^{\prime}(0)=0, \\
u(1) & =\int_{0}^{1} g(s) u(s) d s,
\end{aligned}
$$

where $2<\alpha \leqslant 3$. By using the monotone iteration method and some inequalities technique, the existence result of positive solutions is obtained.

By the same method, Zhang [18] discussed the following fractional differential equation with Riemann-Stieltjes integral boundary conditions:

$$
\begin{aligned}
D_{0+}^{\alpha} u(t)+f(t, u(t)) & =0, \quad t \in(0,1), \\
u(0) & =u^{\prime}(0)=0, \\
D_{0+}^{\beta} u(1) & =\int_{0}^{1} D_{0+}^{\beta} u(t) d A(t),
\end{aligned}
$$

where $2<\alpha \leqslant 3,0 \leqslant \beta \leqslant 1, A(t)$ is a bounded variation, and $\int_{0}^{1} D_{0+}^{\beta} u(t) d A(t)$ denotes a Riemann-Stieltjes integral with 
a signed measure. This includes both the multipoint and a Riemann integral in a single framework.

Motivated by the wide applications of nonlocal boundary value problems and the results mentioned above, we consider the following fractional differential equation with multistrip Riemann-Stieltjes integral boundary conditions:

$$
\begin{aligned}
D_{0+}^{\alpha} u(t)+f\left(t, u(t), D_{0+}^{\beta} u(t)\right) & =0, \quad t \in(0,1), \\
u(0) & =D_{0+}^{\beta} u(0)=0, \\
u(1) & =\sum_{i=1}^{m} \alpha_{i} \int_{I_{i}} u(t) d A(t),
\end{aligned}
$$

where $2<\alpha \leqslant 3,0<\beta<1, I_{i} \subset(0,1), i=1,2, \ldots, m$, and $D_{0+}^{\alpha}$ is the standard Riemann-Liouville derivative; the nonlinear term $f$ is related to the lower derivative of the function $u$. We emphasize that multistrip integral boundary conditions in (5) state that the value of unknown function at the right end point $t=1$ of the given interval is equal to the linear combination of the Riemann-Stieltjes integral values of the unknown function on the subinterval $I_{i}$, for $i=1,2, \ldots, m$. The consideration of the fractional differential equation together with multistrip Riemann-Stieltjes integral boundary conditions makes problem (4) and (5) new. The proof of our main results is based on the Leggett-Williams fixed point theorem in a cone, which we present now.

Theorem 1 (Leggett-Williams fixed point theorem). Let $P$ be a cone in a real Banach space $E, P_{c}=\{x \in P \mid\|x\| \leqslant c\}$, $\Psi$ be a nonnegative continuous concave functional on $P$ such that $\Psi(x) \leqslant\|x\|$ for all $x \in \bar{P}_{c}$, and $P(\Psi, b, d)=\{x \in P \mid b \leqslant$ $\Psi(x),\|x\| \leqslant d\}$. Suppose that $T: \bar{P}_{c} \rightarrow \bar{P}_{c}$ is completely continuous and there exist constants $0<a<b<d \leqslant c$ such that

$\left(S_{1}\right)\{x \in P(\Psi, b, d) \mid \Psi(x)>b\} \neq \varnothing$, and $\Psi(T x)>b$ for $x \in P(\Psi, b, d)$;

$\left(S_{2}\right)\|T x\|<a$ for $\|x\| \leqslant a$;

$\left(S_{3}\right) \Psi(T x)>b$ for $x \in P(\Psi, b, c)$, with $\|T x\|>d$.

Then $T$ has at least three fixed points $x_{1}, x_{2}$, and $x_{3}$, which satisfy

$$
\begin{gathered}
\left\|x_{1}\right\|<a, \\
b<\Psi\left(x_{2}\right), \\
a<\left\|x_{3}\right\| \quad \text { with } \Psi\left(x_{3}\right)<b .
\end{gathered}
$$

If there holds $d=c$, then condition $\left(S_{1}\right)$ implies condition $\left(S_{3}\right)$ of Theorem 1 . Throughout this paper, we always make the following assumptions:

$\left(H_{1}\right) f:[0,1] \times[0,+\infty) \times \mathbb{R} \rightarrow[0,+\infty)$ is continuous;

$\left(H_{2}\right) 2<\alpha \leqslant 3,0<\beta<1,1<\alpha-\beta<2$;

$\left(H_{3}\right) \alpha_{i} \in[0,+\infty), I_{i} \subset(0,1), i=1,2, \ldots, m$, and $A$ : $[0,1] \rightarrow \mathbb{R}$ is an increasing function of bounded variation;

$\left(H_{4}\right) 0<\delta_{1}<1$, where $\delta_{1}=\sum_{i=1}^{m} \alpha_{i} \int_{I_{i}} t^{\alpha-1} d A(t)$.

\section{Preliminaries}

In this section, we will present several definitions and lemmas that are necessary for the proof of our main results.

Definition 2 (see [1]). The Riemann-Liouville fractional integral of order $\alpha>0$ of a function $y:(0, \infty) \rightarrow \mathbb{R}$ is given by

$$
I_{0+}^{\alpha} y(t)=\frac{1}{\Gamma(\alpha)} \int_{0}^{t}(t-s)^{\alpha-1} y(s) d s,
$$

provided the right side is pointwise defined on $[0, \infty)$.

Definition 3 (see [1]). The Riemann-Liouville fractional derivative of order $\alpha>0$ of a function $y:(0, \infty) \rightarrow \mathbb{R}$ is given by

$$
D_{0+}^{\alpha} y(t)=\frac{1}{\Gamma(n-\alpha)}\left(\frac{d}{d t}\right)^{n} \int_{0}^{t}(t-s)^{n-\alpha-1} y(s) d s,
$$

where $n=[\alpha]+1,[\alpha]$ denotes the integer part of number $\alpha$, provided the right side is pointwise defined on $[0, \infty)$.

From the definitions of Riemann-Liouville's derivative, we can obtain the following statement.

Lemma 4. Let $\alpha>0$; if we assume $u \in C(0,1) \cap L^{1}(0,1)$, then the fractional differential equation

$$
D_{0+}^{\alpha} u(t)=0
$$

has $u(t)=C_{1} t^{\alpha-1}+C_{2} t^{\alpha-2}+\cdots+C_{N} t^{\alpha-N}$, for some $C_{i} \in \mathbb{R}, i=$ $1,2, \ldots, N$, as a unique solution, where $N$ is the smallest integer greater than or equal to $\alpha$.

Lemma 5. Let $\alpha>0$; if we assume $u \in C(0,1) \cap L(0,1)$, then

$$
\begin{aligned}
I_{0+}^{\alpha} D_{0+}^{\alpha} u(t)= & u(t)+C_{1} t^{\alpha-1}+C_{2} t^{\alpha-2}+\cdots \\
& +C_{N} t^{\alpha-N},
\end{aligned}
$$

for some $C_{i} \in \mathbb{R}, i=1,2, \ldots, N$, where $N$ is the smallest integer greater than or equal to $\alpha$.

Remark 6. The following properties are useful for our discussion:

(1) $I_{0+}^{\alpha} I_{0+}^{\beta} y(t)=I_{0+}^{\alpha+\beta} y(t)$, for $\alpha>0, \beta>0, y(t) \epsilon$ $L^{1}(0,1)$;

(2) $D_{0+}^{\alpha} I_{0+}^{\alpha} y(t)=y(t)$, for $\alpha>0, y(t) \in L^{1}(0,1)$.

Lemma 7. Suppose that $\left(H_{4}\right)$ holds. For $y \in C(0,1) \cap L^{1}(0,1)$, the unique solution of

$$
\begin{aligned}
D_{0+}^{\alpha} u(t)+y(t) & =0, \quad t \in(0,1), \\
u(0) & =D_{0+}^{\beta} u(0)=0, \\
u(1) & =\sum_{i=1}^{m} \alpha_{i} \int_{I_{i}} u(t) d A(t)
\end{aligned}
$$


Discrete Dynamics in Nature and Society

3

is $u(t)=\int_{0}^{1} G(t, s) y(s) d s$, in which

$$
G(t, s)=G_{0}(t, s)+\frac{t^{\alpha-1}}{1-\delta_{1}} \sum_{i=1}^{m} \alpha_{i} \int_{I_{i}} G_{0}(t, s) d A(t),
$$

where

$$
\begin{aligned}
& G_{0}(t, s)=\frac{1}{\Gamma(\alpha)} \\
& \quad \cdot \begin{cases}t^{\alpha-1}(1-s)^{\alpha-1}-(t-s)^{\alpha-1}, & 0 \leqslant s \leqslant t \leqslant 1, \\
t^{\alpha-1}(1-s)^{\alpha-1}, & 0 \leqslant t \leqslant s \leqslant 1 .\end{cases}
\end{aligned}
$$

Proof. In view of Lemma 5, we reduce problem (11) to an equivalent integral equation

$$
u(t)=-I_{0+}^{\alpha} u(t)+C_{1} t^{\alpha-1}+C_{2} t^{\alpha-2}+C_{3} t^{\alpha-3},
$$

where $C_{1}, C_{2}, C_{3} \in \mathbb{R}$ are arbitrary constants. Consequently the general solution of the problem (11) can be written as

$$
\begin{aligned}
u(t)= & -\frac{1}{\Gamma(\alpha)} \int_{0}^{t}(t-s)^{\alpha-1} y(s) d s+C_{1} t^{\alpha-1}+C_{2} t^{\alpha-2} \\
& +C_{3} t^{\alpha-3} .
\end{aligned}
$$

By $u(0)=D_{0+}^{\beta} u(0)=0$, we find $C_{2}=C_{3}=0$. Set $t=1$ in (15), then

$$
u(1)=-\frac{1}{\Gamma(\alpha)} \int_{0}^{1}(1-s)^{\alpha-1} y(s) d s+C_{1} .
$$

Together with the boundary condition $u(1)=$ $\sum_{i=1}^{m} \alpha_{i} \int_{I_{i}} u(t) d A(t)$, we have

$$
\begin{aligned}
\sum_{i=1}^{m} \alpha_{i} \int_{I_{i}} u(t) d A(t)= & -\frac{1}{\Gamma(\alpha)} \int_{0}^{1}(1-s)^{\alpha-1} y(s) d s \\
& +C_{1} .
\end{aligned}
$$

$$
\begin{aligned}
u(t)= & -\frac{1}{\Gamma(\alpha)} \int_{0}^{t}(t-s)^{\alpha-1} y(s) d s \\
& +\frac{t^{\alpha-1}}{\Gamma(\alpha)} \int_{0}^{1}(1-s)^{\alpha-1} y(s) d s \\
& +t^{\alpha-1} \sum_{i=1}^{m} \alpha_{i} \int_{I_{i}} u(t) d A(t) \\
= & \int_{0}^{1} G_{0}(t, s) y(s) d s+t^{\alpha-1} \sum_{i=1}^{m} \alpha_{i} \int_{I_{i}} u(t) d A(t) .
\end{aligned}
$$

Furthermore,

$$
\begin{aligned}
\sum_{i=1}^{m} \alpha_{i} \int_{I_{i}} u(t) d A(t) & \\
= & \sum_{i=1}^{m} \alpha_{i} \int_{I_{i}} \int_{0}^{1} G_{0}(t, s) y(s) d s d A(t) \\
& +\left(\sum_{i=1}^{m} \alpha_{i} \int_{I_{i}} t^{\alpha-1} d A(t)\right)\left(\sum_{i=1}^{m} \alpha_{i} \int_{I_{i}} u(t) d A(t)\right) .
\end{aligned}
$$

Then

$$
\begin{aligned}
& \sum_{i=1}^{m} \alpha_{i} \int_{I_{i}} u(t) d A(t) \\
& \quad=\frac{1}{1-\delta_{1}} \sum_{i=1}^{m} \alpha_{i} \int_{I_{i}} \int_{0}^{1} G_{0}(t, s) y(s) d s d A(t) .
\end{aligned}
$$

Hence, the solution of (11) is

$$
\begin{aligned}
& u(t)=\int_{0}^{1} G_{0}(t, s) y(s) d s+\frac{t^{\alpha-1}}{1-\delta_{1}} \sum_{i=1}^{m} \alpha_{i} \\
& \cdot \int_{I_{i}} \int_{0}^{1} G_{0}(t, s) y(s) d s d A(t)=\int_{0}^{1} G_{0}(t, s) \\
& \cdot y(s) d s+\frac{t^{\alpha-1}}{1-\delta_{1}} \sum_{i=1}^{m} \alpha_{i} \\
& \cdot \int_{0}^{1} \int_{I_{i}} G_{0}(t, s) y(s) d A(t) d s \\
& =\int_{0}^{1}\left[G_{0}(t, s)+\frac{t^{\alpha-1}}{1-\delta_{1}} \sum_{i=1}^{m} \alpha_{i} \int_{I_{i}} G_{0}(t, s) d A(t)\right] \\
& \cdot y(s) d s=\int_{0}^{1} G(t, s) y(s) d s .
\end{aligned}
$$

Lemma 8. The function $G_{0}(t, s)$ defined by (13) satisfies the following inequality:

$$
\frac{t^{\alpha-1}(1-s)^{\alpha-1}(1-t) s}{\Gamma(\alpha)} \leqslant G_{0}(t, s) \leqslant \frac{s(1-s)^{\alpha-1}}{\Gamma(\alpha-1)},
$$

for $t, s \in[0,1]$.

Proof. For $0 \leqslant s \leqslant t \leqslant 1$, we have $1-s \geqslant 1-t$, and then

$$
\begin{aligned}
G_{0} & (t, s)=\frac{1}{\Gamma(\alpha)}\left[t^{\alpha-1}(1-s)^{\alpha-1}-(t-s)^{\alpha-1}\right] \\
& =\frac{(\alpha-1)}{\Gamma(\alpha)} \int_{t-s}^{t-t s} x^{\alpha-2} d x \\
& \leqslant \frac{(t-t s)^{\alpha-2}[(t-t s)-(t-s)]}{\Gamma(\alpha-1)} \\
& =\frac{t^{\alpha-2}(1-s)^{\alpha-2}(1-t) s}{\Gamma(\alpha-1)} \leqslant \frac{s(1-s)^{\alpha-1}}{\Gamma(\alpha-1)}, \\
G_{0} & (t, s)=\frac{1}{\Gamma(\alpha)}\left[t^{\alpha-1}(1-s)^{\alpha-1}-(t-s)^{\alpha-1}\right] \\
& =\frac{1}{\Gamma(\alpha)}\left[(t-t s)^{\alpha-2}(t-t s)-(t-s)^{\alpha-2}(t-s)\right] \\
& \geqslant \frac{1}{\Gamma(\alpha)}\left[(t-t s)^{\alpha-2}(t-t s)-(t-t s)^{\alpha-2}(t-s)\right] \\
& =\frac{1}{\Gamma(\alpha)}\left[t^{\alpha-2}(1-s)^{\alpha-2}(1-t) s\right] \\
& \geqslant \frac{1}{\Gamma(\alpha)}\left[t^{\alpha-1}(1-s)^{\alpha-1}(1-t) s\right] .
\end{aligned}
$$


For $0 \leqslant t \leqslant s \leqslant 1$, since $2<\alpha \leqslant 3$, we have

$$
\begin{aligned}
G_{0}(t, s) & =\frac{t^{\alpha-1}(1-s)^{\alpha-1}}{\Gamma(\alpha)} \leqslant \frac{(\alpha-1) t^{\alpha-2} t(1-s)^{\alpha-1}}{\Gamma(\alpha)} \\
& \leqslant \frac{t^{\alpha-2} s(1-s)^{\alpha-1}}{\Gamma(\alpha-1)} \leqslant \frac{s(1-s)^{\alpha-1}}{\Gamma(\alpha-1)}, \\
G_{0}(t, s) & =\frac{t^{\alpha-1}(1-s)^{\alpha-1}}{\Gamma(\alpha)} \geqslant \frac{t^{\alpha-1}(1-s)^{\alpha-1}(1-t) s}{\Gamma(\alpha)} .
\end{aligned}
$$

Then the proof is completed.

For convenience, denote

$$
\begin{aligned}
\delta_{2} & =\sum_{i=1}^{m} \alpha_{i} \int_{I_{i}} d A(t), \\
\delta_{3} & =\sum_{i=1}^{m} \alpha_{i} \int_{I_{i}} t^{\alpha} d A(t), \\
G(s) & =\frac{\left(1-\delta_{1}+\delta_{2}\right) s(1-s)^{\alpha-1}}{\left(1-\delta_{1}\right) \Gamma(\alpha-1)}, \text { for } s \in[0,1], \\
\Lambda(s) & =\frac{\left(1-\delta_{1}\right)(1-s)^{\alpha-\beta-1}+\delta_{2}(1-s)^{\alpha-1}}{\Gamma(\alpha-\beta)\left(1-\delta_{1}\right)}, \\
\lambda & =\frac{\theta^{\alpha}\left(1-\delta_{1}\right)+\theta^{\alpha-1}\left(\delta_{1}-\delta_{3}\right)}{\left(1-\delta_{1}+\delta_{2}\right)(\alpha-1)}, \\
\text { where } \theta \in\left(0, \frac{1}{2}\right) & \text { is aconstant. }
\end{aligned}
$$

$\delta_{1}$ is introduced $\left(H_{4}\right)$. It is obvious that $\delta_{2}>\delta_{1}>\delta_{3}, \lambda>0$ and $G(s)>0$ for $s \in(0,1)$.

The following properties of the Green function $G(t, s)$ play an important role in this paper.

Lemma 9. The Green function $G(t, s)$ defined by (12) satisfies the following properties:

(1) $G(t, s)$ is continuous on $[0,1] \times[0,1]$;

(2) $G(t, s) \geqslant 0$ for $t, s \in[0,1]$;

(3) $G(t, s) \leqslant G(s)$ for $t, s \in[0,1]$;

(4) $G(t, s) \geqslant \lambda G(s)$ for $s \in[0,1], t \in[\theta, 1-\theta]$;

(5) $\left|D_{0+}^{\beta} G(t, s)\right| \leqslant \Lambda(s)$ for $t, s \in[0,1]$.

Proof. (1) and (2) hold obviously; we only show that (3)-(5) are true.

(3) For any $t, s \in[0,1]$, by (12), (13), and the right inequality of (22), we get

$$
\begin{aligned}
G(t, s) & =G_{0}(t, s)+\frac{t^{\alpha-1}}{1-\delta_{1}} \sum_{i=1}^{m} \alpha_{i} \int_{I_{i}} G_{0}(t, s) d A(t) \\
& \leqslant \frac{s(1-s)^{\alpha-1}}{\Gamma(\alpha-1)}
\end{aligned}
$$

$$
\begin{aligned}
& +\frac{t^{\alpha-1}}{1-\delta_{1}} \sum_{i=1}^{m} \alpha_{i} \int_{I_{i}} \frac{s(1-s)^{\alpha-1}}{\Gamma(\alpha-1)} d A(t) \\
= & \frac{s(1-s)^{\alpha-1}}{\Gamma(\alpha-1)}+\frac{t^{\alpha-1} s(1-s)^{\alpha-1} \delta_{2}}{\left(1-\delta_{1}\right) \Gamma(\alpha-1)} \\
\leqslant & \frac{\left(1-\delta_{1}+\delta_{2}\right) s(1-s)^{\alpha-1}}{\left(1-\delta_{1}\right) \Gamma(\alpha-1)}=G(s) .
\end{aligned}
$$

(4) For any $s \in[0,1]$, by (12), (13), and the left inequality of (22), we get

$$
\begin{aligned}
& \min _{t \in[\theta, 1-\theta]} G(t, s)=\min _{t \in[\theta, 1-\theta]}\left\{G_{0}(t, s)\right. \\
& \left.+\frac{t^{\alpha-1}}{1-\delta_{1}} \sum_{i=1}^{m} \alpha_{i} \int_{I_{i}} G_{0}(t, s) d A(t)\right\} \\
& \geqslant \min _{t \in[\theta, 1-\theta]}\left\{\frac{t^{\alpha-1}(1-t) s(1-s)^{\alpha-1}}{\Gamma(\alpha)}\right. \\
& \left.+\frac{t^{\alpha-1}}{1-\delta_{1}} \sum_{i=1}^{m} \alpha_{i} \int_{I_{i}} \frac{t^{\alpha-1}(1-t) s(1-s)^{\alpha-1}}{\Gamma(\alpha)} d A(t)\right\} \\
& \geqslant \frac{\theta^{\alpha-1} \theta s(1-s)^{\alpha-1}}{\Gamma(\alpha)}+\frac{\theta^{\alpha-1}}{1-\delta_{1}} \sum_{i=1}^{m} \alpha_{i} \\
& \int_{I_{i}} \frac{\left(t^{\alpha-1}-t^{\alpha}\right) s(1-s)^{\alpha-1}}{\Gamma(\alpha)} d A(t) \\
& =\frac{s(1-s)^{\alpha-1}}{\Gamma(\alpha)}\left[\theta^{\alpha}+\frac{\theta^{\alpha-1}\left(\delta_{1}-\delta_{3}\right)}{1-\delta_{1}}\right]=\lambda G(s) \text {. }
\end{aligned}
$$

(5) By the definition of $G_{0}(t, s)$ and $D_{0+}^{\beta} t^{\mu}=(\Gamma(\mu+1) /$ $\Gamma(\mu-\beta+1)) t^{\mu-\beta}(\mu>-1)$, we have

$$
\begin{aligned}
D_{0+}^{\beta} G_{0}(t, s)=\frac{1}{\Gamma(\alpha-\beta)} & \\
& \cdot \begin{cases}t^{\alpha-\beta-1}(1-s)^{\alpha-1}-(t-s)^{\alpha-\beta-1}, & 0 \leqslant s \leqslant t \leq 1, \\
t^{\alpha-\beta-1}(1-s)^{\alpha-1}, & 0 \leqslant t \leqslant s \leq 1 .\end{cases}
\end{aligned}
$$

Therefore,

$$
\begin{aligned}
& \left|D_{0+}^{\beta} G_{0}(t, s)\right|=\frac{1}{\Gamma(\alpha-\beta)} \\
& . \begin{cases}\left|t^{\alpha-\beta-1}(1-s)^{\alpha-1}-(t-s)^{\alpha-\beta-1}\right|, & 0 \leqslant s \leqslant t \leqslant 1, \\
t^{\alpha-\beta-1}(1-s)^{\alpha-1}, & 0 \leqslant t \leqslant s \leqslant 1\end{cases} \\
& \leqslant \frac{1}{\Gamma(\alpha-\beta)} \\
& \quad \begin{cases}\max \left\{t^{\alpha-\beta-1}(1-s)^{\alpha-1},(t-s)^{\alpha-\beta-1}\right\}, & 0 \leqslant s \leqslant t \leqslant 1, \\
(1-s)^{\alpha-1}, & 0 \leqslant t \leqslant s \leqslant 1\end{cases}
\end{aligned}
$$




$$
\begin{aligned}
& \leqslant \frac{1}{\Gamma(\alpha-\beta)} \\
& \begin{cases}\max \left\{(1-s)^{\alpha-1},(1-s)^{\alpha-\beta-1}\right\}, & 0 \leqslant s \leqslant t \leqslant 1, \\
(1-s)^{\alpha-1}, & 0 \leqslant t \leqslant s \leqslant 1\end{cases} \\
& \leqslant \frac{1}{\Gamma(\alpha-\beta)}(1-s)^{\alpha-\beta-1} .
\end{aligned}
$$

From (13), it is evident that

$$
\left|G_{0}(t, s)\right|=G_{0}(t, s) \leqslant \frac{1}{\Gamma(\alpha)}(1-s)^{\alpha-1} .
$$

It follows from (29) and (30) that

$$
\begin{aligned}
& \left|D_{0+}^{\beta} G(t, s)\right| \\
& \leqslant\left|D_{0+}^{\beta} G_{0}(t, s)\right| \\
& \quad+\left|\frac{\Gamma(\alpha) t^{\alpha-\beta-1}}{\Gamma(\alpha-\beta)\left(1-\delta_{1}\right)} \sum_{i=1}^{m} \alpha_{i} \int_{I_{i}} G_{0}(t, s) d A(t)\right| \\
& \leqslant \frac{(1-s)^{\alpha-\beta-1}}{\Gamma(\alpha-\beta)} \\
& \quad+\frac{\Gamma(\alpha)}{\Gamma(\alpha-\beta)\left(1-\delta_{1}\right)} \sum_{i=1}^{m} \alpha_{i} \int_{I_{i}} \frac{(1-s)^{\alpha-1}}{\Gamma(\alpha)} d A(t) \\
& =\frac{\left(1-\delta_{1}\right)(1-s)^{\alpha-\beta-1}+\delta_{2}(1-s)^{\alpha-1}}{\Gamma(\alpha-\beta)\left(1-\delta_{1}\right)}=\Lambda(s) .
\end{aligned}
$$

The proof of the Lemma is completed.

\section{Existence Result}

Define the space $E=\left\{u(t) \mid u(t) \in C[0,1]\right.$ and $D_{0+}^{\beta} u(t) \in$ $C[0,1]\}$ is endowed with the ordering $u \leqslant v$ if $u(t) \leqslant$ $v(t)$, for all $t \in[0,1]$, and endowed with the norm $\|u\|=$ $\max \left\{\|u\|_{0},\left\|D_{0+}^{\beta} u\right\|_{0}\right\}$, where $\|u\|_{0}=\max _{t \in[0,1]}|u(t)|$.

Lemma 10. $(E,\|\cdot\|)$ is a Banach space.

Proof. Let $\left\{u_{n}\right\}_{n=1}^{\infty}$ be a Cauchy sequence in the space $(E,\|\cdot\|)$. Then clearly $\left\{u_{n}\right\}_{n=1}^{\infty}$ and $\left\{D_{0+}^{\beta} u_{n}\right\}_{n=1}^{\infty}$ are Cauchy sequences in the space $C[0,1]$. Therefore, $\left\{u_{n}\right\}_{n=1}^{\infty}$ and $\left\{D_{0+}^{\beta} u_{n}\right\}_{n=1}^{\infty}$ converge to some $v$ and $w$ on $[0,1]$ uniformly and $v, w \in C[0,1]$. We need to proof that $w=D_{0+}^{\beta} v$.

Note that

$$
\begin{aligned}
& \left|I_{0+}^{\beta} D_{0+}^{\beta} u_{n}(t)-I_{0+}^{\beta} w(t)\right| \\
& \quad \leqslant \frac{1}{\Gamma(\beta)} \int_{0}^{t}(t-s)^{\beta-1}\left|D_{0+}^{\beta} u_{n}(s)-w(s)\right| d s \\
& \quad \leqslant \frac{1}{\Gamma(\beta+1)} \max _{s \in[0,1]}\left|D_{0+}^{\beta} u_{n}(s)-w(s)\right| .
\end{aligned}
$$

By the convergence of $\left\{D_{0+}^{\beta} u_{n}\right\}_{n=1}^{\infty}$, we have

$$
\lim _{n \rightarrow \infty} I_{0+}^{\beta} D_{0+}^{\beta} u_{n}(t)=I_{0+}^{\beta} w(t)
$$

uniformly for $t \in[0,1]$. On the other hand, by Lemma 5 one has $I_{0+}^{\beta} D_{0+}^{\beta} u_{n}(t)=u_{n}(t)+C_{1} t^{\beta-1}$, for $t \in[0,1]$ and some $C_{1} \in \mathbb{R}$. Further, we can obtain

$$
\begin{aligned}
\lim _{n \rightarrow \infty} I_{0+}^{\beta} D_{0+}^{\beta} u_{n}(t) & =\lim _{n \rightarrow \infty} u_{n}(t)+C_{1} t^{\beta-1} \\
& =v(t)+C_{1} t^{\beta-1} .
\end{aligned}
$$

From (33) and (34), we have

$$
I_{0+}^{\beta} w(t)=v(t)+C_{1} t^{\beta-1}, \quad \text { for } t \in[0,1] .
$$

Taking the $\beta$-order derivative on both sides of (35) yields

$$
D_{0+}^{\beta} I_{0+}^{\beta} w(t)=D_{0+}^{\beta}\left[v(t)+C_{1} t^{\beta-1}\right], \quad \text { for } t \in[0,1] \text {. }
$$

In view of Remark 6 and Lemma 4, we find that

$$
w(t)=D_{0+}^{\beta} v(t), \quad \text { for } t \in[0,1] .
$$

This completes the proof.

Define the cone $P \subset E$ by

$$
P=\{u \in E \mid u(t) \geqslant 0\} .
$$

Let the nonnegative continuous concave functional $\Psi$ on the cone $P$ be defined by

$$
\Psi(u)=\min _{\theta \leqslant t \leqslant 1-\theta}|u(t)|
$$

Lemma 11. Assume conditions $\left(H_{1}\right)-\left(H_{4}\right)$ hold. For any $u \in E$, define the operator $T$ by

$$
(T u)(t)=\int_{0}^{1} G(t, s) f\left(s, u(s), D_{0+}^{\beta} u(s)\right) d s,
$$

$$
0 \leqslant t \leqslant 1 \text {, }
$$

and then $T: P \rightarrow P$ is completely continuous.

Proof. First, we prove that $T: P \rightarrow P$. In view of the nonnegativeness and continuity of $G(t, s)$ and $f\left(t, u(t), D_{0+}^{\beta} u(t)\right), T$ is continuous and $(T u)(t) \geqslant 0$ for $u \in P$. Hence $T P \subset P$.

Next, we show $T$ is uniformly bounded. Let $\Omega \subset P$ be bounded; that is, there exists a positive constant $M>0$ such that $\|u\| \leqslant M$, for all $u \in \Omega$. Let $L=1+\max \{f(t$, $\left.u(t), D_{0+}^{\beta} u(t)\right) \mid 0 \leqslant t \leqslant 1,0 \leqslant u \leqslant M,-M \leqslant D_{0+}^{\beta} u(t) \leqslant$ $M\}$; then for $u \in \Omega$, from the Lemma 9, we have

$$
\begin{aligned}
& |(T u)(t)|=\int_{0}^{1} G(t, s) f\left(s, u(s), D_{0+}^{\beta} u(s)\right) d s \\
& \quad \leqslant L \int_{0}^{1} G(s) d s,
\end{aligned}
$$




$$
\begin{aligned}
& \left|D_{0+}^{\beta}(\mathrm{Tu})(t)\right| \\
& \quad=\left|\int_{0}^{1} D_{0+}^{\beta} G(t, s) f\left(s, u(s), D_{0+}^{\beta} u(s)\right) d s\right| \\
& \quad \leqslant L \int_{0}^{1}\left|D_{0+}^{\beta} G(t, s)\right| d s \leqslant L \int_{0}^{1} \Lambda(s) d s .
\end{aligned}
$$

Hence, $T(\Omega)$ is bounded.

Finally, we show $T$ is equicontinuous. Indeed, for any $u \in$ $\Omega, t_{1}, t_{2} \in[0,1], t_{1}<t_{2}$, we have

$$
\begin{aligned}
& \left|(T u)\left(t_{2}\right)-(T u)\left(t_{1}\right)\right| \leqslant \int_{0}^{1}\left|G\left(t_{2}, s\right)-G\left(t_{1}, s\right)\right| \\
& \cdot f\left(s, u(s), D_{0+}^{\beta} u(s)\right) d s \leqslant L \int_{0}^{1} \mid G_{0}\left(t_{2}, s\right) \\
& -G_{0}\left(t_{1}, s\right) \\
& +\frac{\left(t_{2}^{\alpha-1}-t_{1}^{\alpha-1}\right)}{1-\delta_{1}} \sum_{i=1}^{m} \alpha_{i} \int_{I_{i}} G_{0}(t, s) d A(t) \mid d s \\
& \quad=L \int_{0}^{1} \mid \frac{1}{\Gamma(\alpha)}\left(t_{2}^{\alpha-1}-t_{1}^{\alpha-1}\right)(1-s)^{\alpha-1} \\
& -\frac{1}{\Gamma(\alpha)}\left[\left(t_{2}-s\right)^{\alpha-1}-\left(t_{1}-s\right)^{\alpha-1}\right] \\
& +\frac{\left(t_{2}^{\alpha-1}-t_{1}^{\alpha-1}\right)}{1-\delta_{1}} \sum_{i=1}^{m} \alpha_{i} \int_{I_{i}} G_{0}(t, s) d A(t) \mid d s \\
& \quad \leqslant \frac{L}{\Gamma(\alpha)} \int_{0}^{1}\left|\left(t_{2}^{\alpha-1}-t_{1}^{\alpha-1}\right)(1-s)^{\alpha-1}\right| d s+\frac{L}{\Gamma(\alpha)} \\
& \quad \cdot \int_{0}^{1}\left|\left(t_{2}-s\right)^{\alpha-1}-\left(t_{1}-s\right)^{\alpha-1}\right| d s+\frac{L}{1-\delta_{1}} \\
& \cdot \int_{0}^{1}\left|t_{2}^{\alpha-1}-t_{1}^{\alpha-1}\right| \sum_{i=1}^{m} \alpha_{i} \int_{I_{i}} G_{0}(t, s) d A(t) d s .
\end{aligned}
$$

Note that, applying the mean value theorem, we arrive at $t_{2}^{\alpha-1}-t_{1}^{\alpha-1}<(\alpha-1)\left(t_{2}-t_{1}\right)$ and $\left(t_{2}-s\right)^{\alpha-1}-\left(t_{1}-s\right)^{\alpha-1}<$ $(\alpha-1)\left(t_{2}-t_{1}\right)$, which implies that

$$
\begin{aligned}
& \left|(T u)\left(t_{2}\right)-(T u)\left(t_{1}\right)\right|<L(\alpha-1)\left[\frac{2}{\Gamma(\alpha)}\right. \\
& \left.+\frac{1}{1-\delta_{1}} \max _{0 \leqslant s \leqslant 1}\left\{\sum_{i=1}^{m} \alpha_{i} \int_{I_{i}} G_{0}(t, s) d A(t)\right\}\right]\left(t_{2}-t_{1}\right) \\
& \longrightarrow 0, \quad \text { as } t_{2} \longrightarrow t_{1} .
\end{aligned}
$$

Moreover,

$$
\begin{gathered}
\left|D_{0+}^{\beta}(T u)\left(t_{2}\right)-D_{0+}^{\beta}(T u)\left(t_{1}\right)\right| \leqslant \int_{0}^{1} \mid D_{0+}^{\beta} G\left(t_{2}, s\right) \\
-D_{0+}^{\beta} G\left(t_{1}, s\right) \mid f\left(s, u(s), D_{0+}^{\beta} u(s)\right) d s
\end{gathered}
$$

$$
\begin{aligned}
& \leqslant L \int_{0}^{1} \mid D_{0+}^{\beta} G_{0}\left(t_{2}, s\right)-D_{0+}^{\beta} G_{0}\left(t_{1}, s\right) \\
& +\frac{\Gamma(\alpha)\left(t_{2}^{\alpha-\beta-1}-t_{1}^{\alpha-\beta-1}\right)}{\Gamma(\alpha-\beta)\left(1-\delta_{1}\right)} \sum_{i=1}^{m} \alpha_{i} \\
& \cdot \int_{I_{i}} G_{0}(t, s) d A(t) \mid d s \\
& =L \int_{0}^{1} \mid \frac{1}{\Gamma(\alpha-\beta)}\left(t_{2}^{\alpha-\beta-1}-t_{1}^{\alpha-\beta-1}\right)(1-s)^{\alpha-1} \\
& -\frac{1}{\Gamma(\alpha-\beta)}\left[\left(t_{2}-s\right)^{\alpha-\beta-1}-\left(t_{1}-s\right)^{\alpha-\beta-1}\right] \\
& +\frac{\Gamma(\alpha)\left(t_{2}^{\alpha-\beta-1}-t_{1}^{\alpha-\beta-1}\right)}{\Gamma(\alpha-\beta)\left(1-\delta_{1}\right)} \sum_{i=1}^{m} \alpha_{i} \\
& \cdot \int_{I_{i}} G_{0}(t, s) d A(t) \mid d s \leqslant \frac{L}{\Gamma(\alpha-\beta)} \\
& \cdot \int_{0}^{1}\left|\left(t_{2}^{\alpha-\beta-1}-t_{1}^{\alpha-\beta-1}\right)(1-s)^{\alpha-1}\right| d s \\
& +\frac{L}{\Gamma(\alpha-\beta)} \int_{0}^{1}\left|\left(t_{2}-s\right)^{\alpha-\beta-1}-\left(t_{1}-s\right)^{\alpha-\beta-1}\right| d s \\
& +\frac{L \Gamma(\alpha)}{\Gamma(\alpha-\beta)\left(1-\delta_{1}\right)} \int_{0}^{1}\left|t_{2}^{\alpha-\beta-1}-t_{1}^{\alpha-\beta-1}\right| \sum_{i=1}^{m} \alpha_{i} \\
& \cdot \int_{I_{i}} G_{0}(t, s) d A(t) d s<\frac{L}{\Gamma(\alpha-\beta-1)}[2 \\
& \left.+\frac{\Gamma(\alpha)}{1-\delta_{1}} \max _{0 \leqslant s \leqslant 1}\left\{\sum_{i=1}^{m} \alpha_{i} \int_{I_{i}} G_{0}(t, s) d A(t)\right\}\right]\left(t_{2}-t_{1}\right) \\
& \longrightarrow 0, \quad \text { as } t_{2} \longrightarrow t_{1} \text {. }
\end{aligned}
$$

Therefore, (43) and (44) imply that $T: P \rightarrow P$ is equicontinuous for all $u \in \Omega$. By means of the Arzela-Ascoli theorem, $T: P \rightarrow P$ is completely continuous.

For convenience, we denote

$$
\begin{aligned}
& M=\frac{1}{\max \left\{\int_{0}^{1} \Lambda(s) d s, \int_{0}^{1} G(s) d s\right\}}, \\
& N=\frac{1}{\lambda \int_{\theta}^{1-\theta} G(s) d s} .
\end{aligned}
$$

Theorem 12. Assume that conditions $\left(H_{1}\right)-\left(H_{4}\right)$ hold, there exist nonnegative numbers $0<a<b<c \theta$, and $f(t, u, v)$ satisfies the following conditions:

$\left(H_{5}\right) f(t, u, v) \leqslant M c$, for $(t, u, v) \in[0,1] \times[0, c] \times[-c, c] ;$ 
( $\left.H_{6}\right) f(t, u, v)<M a$, for $(t, u, v) \in[0,1] \times[0, a] \times[-a, a]$;

$\left(H_{7}\right) f(t, u, v) \geqslant N b$, for $(t, u, v) \in[\theta, 1-\theta] \times[b, c] \times[-c, c]$.

Then BVP (4) and (5) has at least three positive solutions $u_{1}$, $u_{2}$, and $u_{3}$ such that

$$
\begin{aligned}
\left\|u_{1}\right\| & <a, \\
b & <\Psi\left(u_{2}\right)<\left\|u_{2}\right\| \leqslant c, \\
a & <\left\|u_{3}\right\| \leqslant c \quad \text { with } \Psi\left(u_{3}\right)<b .
\end{aligned}
$$

Proof. We will verity that the conditions $\left(S_{1}\right)-\left(S_{3}\right)$ of Theorem 1 are satisfied.

Let $\bar{P}_{C}=\{u \in E \mid u(t) \geqslant 0$ and $\|u\| \leqslant c\}$. We first prove that $T: \bar{P}_{c} \rightarrow \bar{P}_{c}$ is completely continuous. From Lemma 11, we only need to prove that $T \bar{P}_{c} \subset \bar{P}_{c}$. For any $u \in \bar{P}_{c}$, we have $0 \leqslant u(t) \leqslant c,-c \leqslant D_{0+}^{\beta} u(t) \leqslant c$, for all $t \in[0,1]$. The assumption $\left(H_{5}\right)$ implies $f\left(t, u(t), D_{0+}^{\beta} u(t)\right) \leqslant M c$ for $0 \leqslant t \leqslant 1$. Consequently, for $t \in[0,1]$,

$$
\begin{aligned}
& |(T u)(t)|=\left|\int_{0}^{1} G(t, s) f\left(s, u(s), D_{0+}^{\beta} u(s)\right) d s\right| \\
& \quad \leqslant M c \int_{0}^{1} G(s) d s \leqslant c, \\
& \left|D_{0+}^{\beta}(T u)(t)\right| \\
& \quad=\left|\int_{0}^{1} D_{0+}^{\beta} G(t, s) f\left(s, u(s), D_{0+}^{\beta} u(s)\right) d s\right| \\
& \quad \leqslant M c \int_{0}^{1}\left|D_{0+}^{\beta} G(t, s)\right| d s \leqslant M c \int_{0}^{1} \Lambda(s) d s \leqslant c .
\end{aligned}
$$

Thus, $\|T u\| \leqslant c$ and further to get $T \bar{P}_{c} \subset \bar{P}_{c}$. Therefore $T: \bar{P}_{c} \rightarrow \bar{P}_{c}$ is completely continuous.

Similarly, the conditions $\left(S_{2}\right)$ of Theorem 1 can be obtained by the assumption $\left(H_{6}\right)$. Here we do not do more explanation.

Finally, in order to verity $\{u \in P(\Psi, b, c) \mid \Psi(u)>b\} \neq \varnothing$, we make $u(t)=(b / \theta) t^{\beta}, 0 \leqslant t \leqslant 1$. It is easy to find that

$$
\begin{aligned}
\Psi(u) & =\min _{\theta \leqslant t \leqslant 1-\theta}\left|\frac{b}{\theta} t^{\beta}\right| \geqslant \frac{b}{\theta} \theta^{\beta}>b, \\
\|u\| & =\max \left\{\max _{t \in[0,1]}|u(t)|, \max _{t \in[0,1]}\left|D_{0+}^{\beta} u(t)\right|\right\} \\
& =\max \left\{\frac{b}{\theta}, \Gamma(\beta+1) \frac{b}{\theta}\right\}=\frac{b}{\theta}<c .
\end{aligned}
$$

If $u \in P(\Psi, b, c)$, we have $b \leqslant u(t) \leqslant c,-c \leqslant D_{0+}^{\beta} u(t) \leqslant c$, for $\theta \leqslant t \leqslant 1-\theta$. Then

$$
\begin{aligned}
\Psi(T u) & =\min _{\theta \leqslant t \leqslant 1-\theta}|(T u)(t)| \\
& \geqslant \int_{0}^{1} \lambda G(s) f\left(s, u(s), D_{0+}^{\beta} u(s)\right) d s \\
& >N b \int_{\theta}^{1-\theta} \lambda G(s) d s=b ;
\end{aligned}
$$

that is, $\Psi(T u)>b$ for all $u \in P(\Psi, b, c)$. This shows that condition $\left(S_{1}\right)$ of Theorem 1 is also satisfied.

From the above, BVP (4) and (5) has at least three positive solutions $u_{1}, u_{2}$, and $u_{3}$ such that

$$
\begin{aligned}
\left\|u_{1}\right\| & <a, \\
b & <\Psi\left(u_{2}\right)<\left\|u_{2}\right\| \leqslant c, \\
a & <\left\|u_{3}\right\| \leqslant c \quad \text { with } \Psi\left(u_{3}\right)<b .
\end{aligned}
$$

The proof is completed.

\section{Example}

Here we provide two cases to verify the feasibility and breadth of the conclusion, where the strip intervals in boundary condition (5) satisfy intersection relation and inclusion relation in Examples 1 and 2, respectively.

Example 1. Consider the boundary value problem of nonlinear fractional differential equations as follows:

$$
\begin{aligned}
& D_{0+}^{2.3} u(t)+f\left(t, u(t), D_{0+}^{0.5} u(t)\right)=0, \quad t \in(0,1), \\
& u(0)=D_{0+}^{0.5} u(0)=0, \\
& u(1)=\sum_{i=1}^{2} \alpha_{i} \int_{I_{i}} u(t) d A(t),
\end{aligned}
$$

where $\alpha_{1}=1 / 2, \alpha_{2}=3 / 5, I_{1}=[1 / 8,5 / 8]$, and $I_{2}=$ $[3 / 8,7 / 8]$ satisfy $I_{1} \cap I_{2} \neq \varnothing$ and $I_{1}, I_{2}$ do not contain each other. Let $A(t)=t^{2}+t$ for $t \in[0,1]$ and

$$
f(t, u, v)= \begin{cases}\frac{t}{10}+\frac{|v|}{100}+\frac{5 u^{7}}{9}, & u<3 \\ \frac{t}{10}+\frac{|v|}{100}+1215, & u \geqslant 3\end{cases}
$$

It is easy to see that $f(t, u, v)$ satisfies condition $\left(H_{1}\right)$.

Take $\theta=1 / 10$. By a simple calculation, we obtain $\delta_{1}=$ $0.5202<1, \delta_{2}=1.1125, \delta_{3}=0.3257, \int_{0}^{1} G(s) d s=$ 0.4827, $\int_{0}^{1} \Lambda(s) d s=1.3431, \lambda=0.0059, M=0.7445, N=$ 366.23 .

Set $a=1, b=3, c=2000$ such that $0<a<b<c \theta$, and, in addition,

$$
\begin{aligned}
\left(H_{5}\right) & f(t, u, v) \leqslant 1235.1<1489=M c, \text { for }(t, u, v) \in[0,1] \times \\
& {[0,2000] \times[-2000,2000] ; } \\
\left(H_{6}\right) & f(t, u, v) \leqslant 0.6656<0.7445=M a, \text { for }(t, u, v) \in \\
& {[0,1] \times[0,1] \times[-1,1] ; } \\
\left(H_{7}\right) & f(t, u, v) \geqslant 1215.01>1098.69=N b, \text { for }(t, u, v) \in \\
& {[0.1,0.9] \times[3,2000] \times[-2000,2000] . }
\end{aligned}
$$

Thus, all the conditions are satisfied. According to Theorem 12, BVP (51) has at least three positive solutions $u_{1}, u_{2}$, and $u_{3}$ such that $\left\|u_{1}\right\|<1,3<\min _{t \in[0.1,0.9]} u_{2}(t)<$ $\left\|u_{2}\right\| \leqslant 2000$, and $1<\left\|u_{3}\right\| \leqslant 2000$ with $\min _{t \in[0.1,0.9]} u_{3}(t)<3$. 
Example 2. Consider the boundary value problem of nonlinear fractional differential equations as follows:

$$
\begin{aligned}
& D_{0+}^{2.3} u(t)+f\left(t, u(t), D_{0+}^{0.5} u(t)\right)=0, \quad t \in(0,1) \\
& u(0)=D_{0+}^{0.5} u(0)=0 \\
& u(1)=\sum_{i=1}^{2} \alpha_{i} \int_{I_{i}} u(t) d A(t)
\end{aligned}
$$

where $\alpha_{1}=1 / 2, \alpha_{2}=3 / 5, I_{1}=[1 / 8,7 / 8], I_{2}=[3 / 8,5 / 8]$, and $I_{1} \supset I_{2}$. Let $A(t)=t^{2}+t$ for $t \in[0,1]$,

$$
f(t, u, v)= \begin{cases}\frac{t}{20}+\frac{|v|}{50}+\frac{9 u^{6}}{16}, & u<4 \\ \frac{t}{20}+\frac{|v|}{50}+2304, & u \geqslant 4\end{cases}
$$

It is easy to see that $f(t, u, v)$ satisfies condition $\left(H_{1}\right)$.

Take $\theta=1 / 10$. By a simple calculation, we obtain $\delta_{1}=$ $0.4768<1, \delta_{2}=1.05, \delta_{3}=0.2929, \int_{0}^{1} G(s) d s=0.4414$, $\int_{0}^{1} \Lambda(s) d s=1.5333, \lambda=0.0058, M=0.6522, N=411.18$.

Set $a=1, b=4, c=4000$ such that $0<a<b<c \theta$, and, in addition,

$$
\begin{aligned}
\left(H_{5}\right) & f(t, u, v) \leqslant 2384.05<2608.8=M c, \text { for }(t, u, v) \in \\
& {[0,1] \times[0,4000] \times[-4000,4000] ; } \\
\left(H_{6}\right) & f(t, u, v) \leqslant 0.6325<0.6522=M a, \text { for }(t, u, v) \in \\
& {[0,1] \times[0,1] \times[-1,1] ; } \\
\left(H_{7}\right) & f(t, u, v) \geqslant 2304.005>1644.72=N b, \text { for }(t, u, v) \in \\
& {[0.1,0.9] \times[4,4000] \times[-4000,4000] . }
\end{aligned}
$$

Thus, all the conditions are satisfied. According to Theorem 12, BVP (53) has at least three positive solutions $u_{1}, u_{2}$, and $u_{3}$ such that $\left\|u_{1}\right\|<1,4<\min _{t \in[0.1,0.9]} u_{2}(t)<$ $\left\|u_{2}\right\| \leqslant 4000$, and $1<\left\|u_{3}\right\| \leqslant 4000$ with $\min _{t \in[0.1,0.9]} u_{3}(t)<4$.

\section{Conflicts of Interest}

The authors declare that they have no conflicts of interest.

\section{Acknowledgments}

This work is supported by Chinese Universities Scientific Fund (Project no. 2017LX003).

\section{References}

[1] I. Podlubny, Fractional Differential Equations, vol. 198 of Mathematics in Science and Engineering, Academic Press, New York, NY, USA, 1999.

[2] A. A. Kilbas, H. M. Srivastava, and J. J. Trujillo, Theory and Applications of Fractional Differential Equations, Amsterdam, Elsevier, 2006.

[3] V. Lakshmikantham, S. Leela, and J. V. Devi, Theory of Fractional Dynamic Systems, Cambridge Scientific Publishers, Cambridge, 2009.
[4] D. Baleanu, K. Diethelm, E. Scalas, and J. J. Trujillo, Fractional Calculus Models and Numerical Methods, vol. 3 of Series on Complexity, Nonlinearity and Chaos, World Scientific, 2012.

[5] Z. Bai, "On positive solutions of a nonlocal fractional boundary value problem," Nonlinear Analysis. Theory, Methods \& Applications. An International Multidisciplinary Journal, vol. 72, no. 2, pp. 916-924, 2010.

[6] D. Jiang and C. Yuan, "The positive properties of the Green function for Dirichlet-type boundary value problems of nonlinear fractional differential equations and its application," Nonlinear Analysis. Theory, Methods \& Applications, vol. 72, no. 2, pp. 710-719, 2010.

[7] T. Jankowski, "Boundary problems for fractional differential equations," Applied Mathematics Letters, vol. 28, pp. 14-19, 2014.

[8] L. Lin, X. Liu, and H. Fang, "Method of upper and lower solutions for fractional differential equations," Electronic Journal of Differential Equations, vol. 2012, 2012.

[9] Z. B. Bai, S. Zhang, S. J. Sun, and C. Yin, "Monotone iterative method for a class of fractional differential equations," Electronic Journal of Differential Equations, vol. No. 06, p. 2016, 2016.

[10] X. Zhang and Q. Zhong, "Multiple positive solutions for nonlocal boundary value problems of singular fractional differential equations," Boundary Value Problems, Paper No. 65, 11 pages, 2016.

[11] W. Yang, "Positive solutions for a coupled system of nonlinear fractional differential equations with integral boundary conditions," Computers \& Mathematics with Applications, vol. 63, no. 1, pp. 288-297, 2012.

[12] R. A. Khan, M. ur Rehman, and J. Henderson, "Existence and uniqueness of solutions for nonlinear fractional differential equations with integral boundary conditions," Fractional Differential Calculus, vol. 1, no. 1, pp. 29-43, 2011.

[13] J. Jin, X. Liu, and M. Jia, "Existence of positive solutions for singular fractional differential equations with integral boundary conditions," Electronic Journal of Differential Equations, No. 63, 14 pages, 2012.

[14] M. Feng, X. Zhang, and W. Ge, "New existence results for higher-order nonlinear fractional differential equation with integral boundary conditions," Boundary Value Problems, vol. 2011, Article ID 720702, 20 pages, 2011.

[15] D. Min, L. Liu, and Y. Wu, "Uniqueness of positive solutions for the singular fractional differential equations involving integral boundary value conditions," Boundary Value Problems, 2018:23 pages, 2018.

[16] X. Liu, L. Liu, and Y. Wu, "Existence of positive solutions for a singular nonlinear fractional differential equation with integral boundary conditions involving fractional derivatives," Boundary Value Problems, 2018:24 pages, 2018.

[17] Y. Sun and M. Zhao, "Positive solutions for a class of fractional differential equations with integral boundary conditions," Applied Mathematics Letters, vol. 34, pp. 17-21, 2014.

[18] H.-E. Zhang, "Iterative solutions for fractional nonlocal boundary value problems involving integral conditions," Boundary Value Problems, Paper No. 3, 13 pages, 2016. 


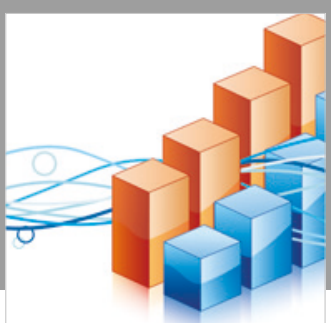

Advances in

Operations Research

\section{-n-m}
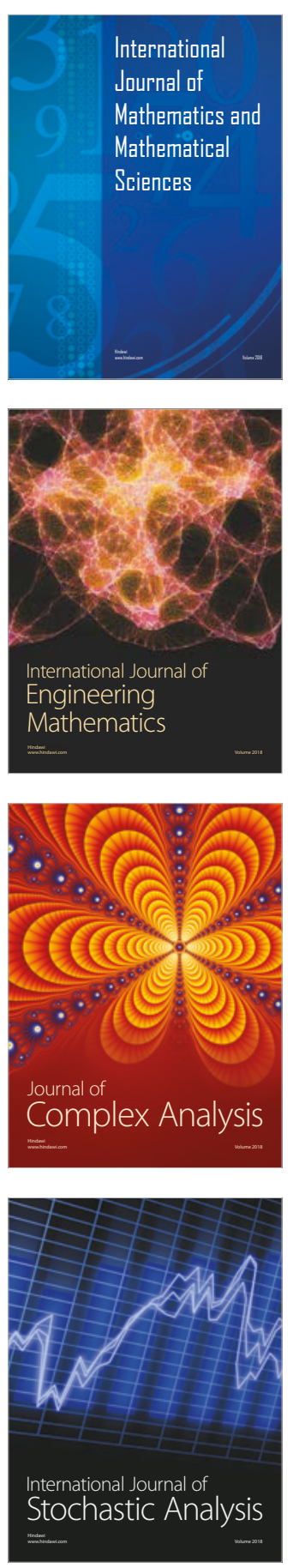
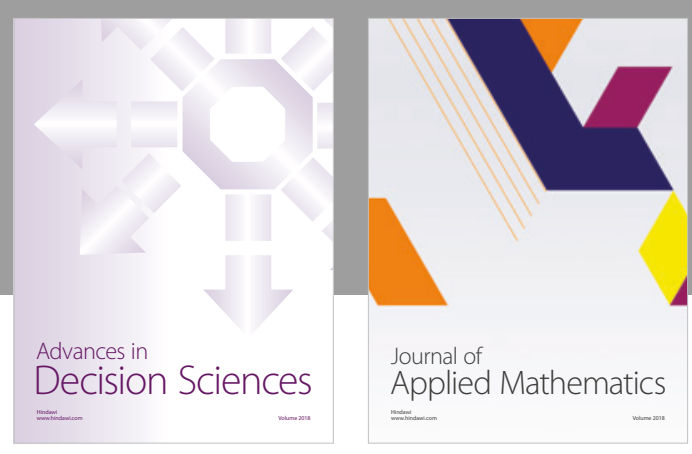

Journal of

Applied Mathematics
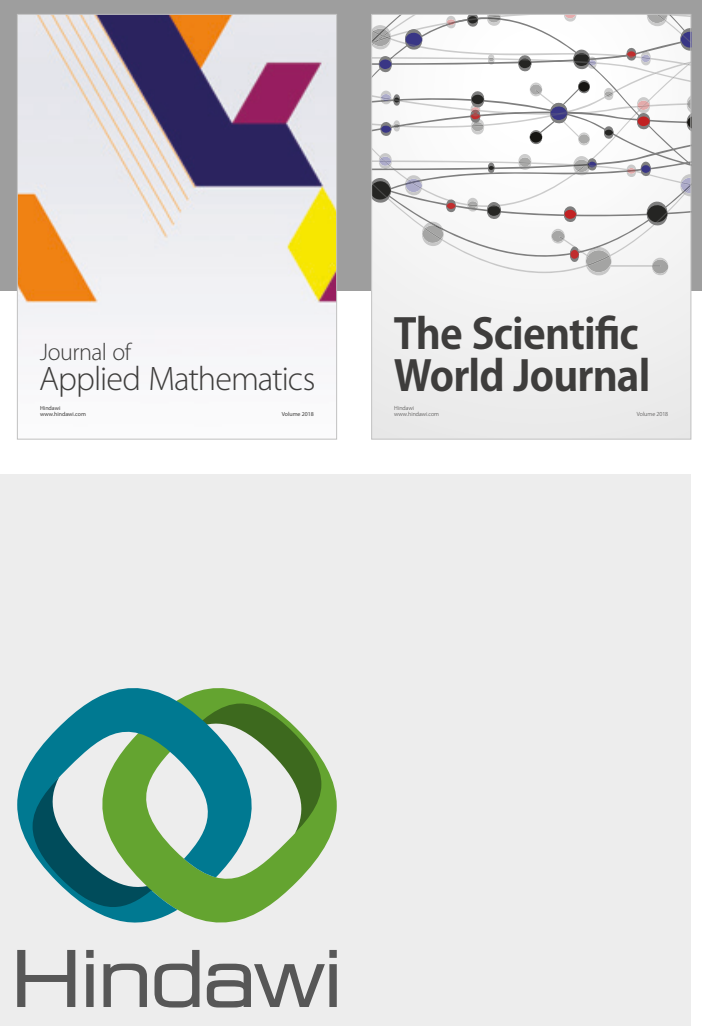

Submit your manuscripts at

www.hindawi.com

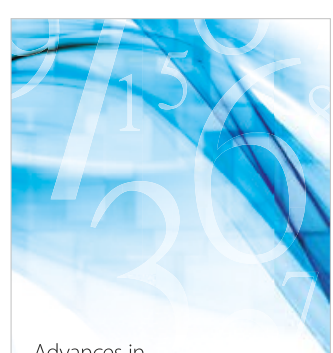

Advances in
Numerical Analysis
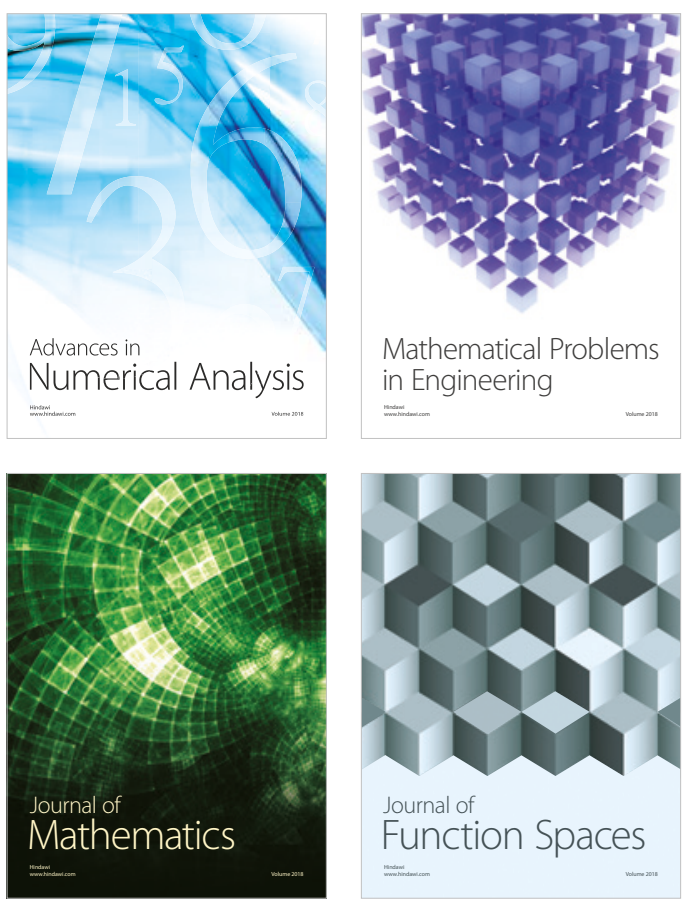

Mathematical Problems in Engineering

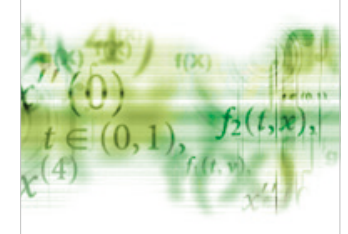

International Journal of

Differential Equations

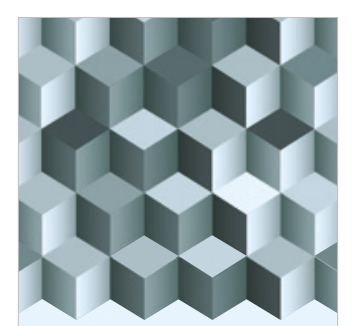

Journal of

Function Spaces

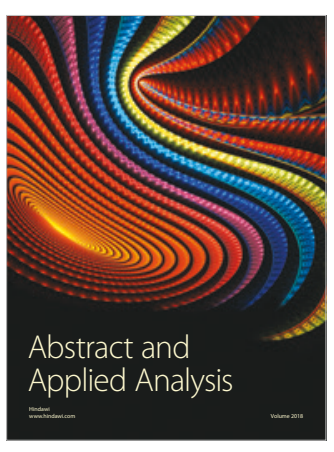

The Scientific

World Journal

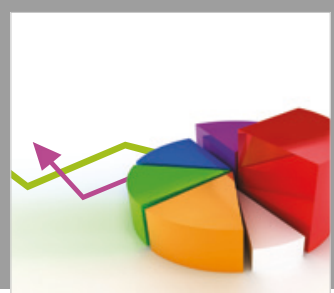

Journal of

Probability and Statistics
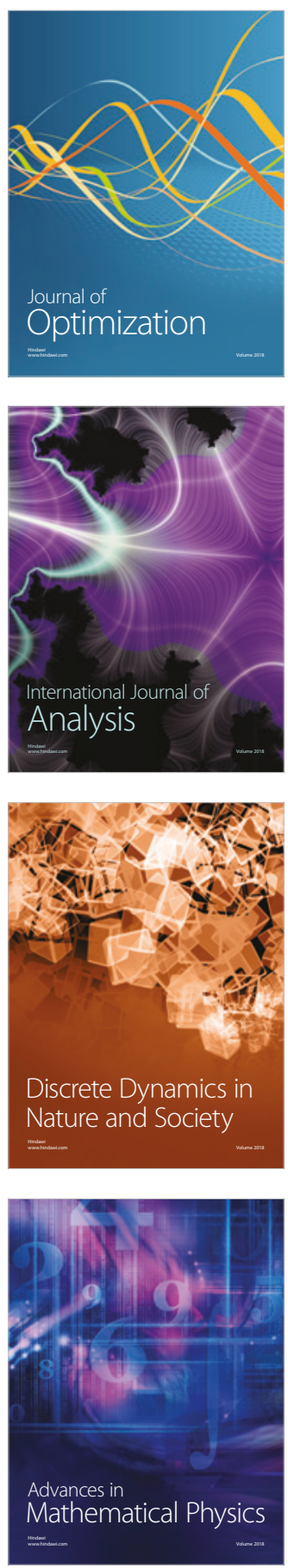